\title{
Les Sources de la Sylviculture de Maclot
}

\section{LES \\ ORDONNANCES DE L'ARCHIDUC ALBERT \\ ET DE \\ L'INFANTE ISABELLE-CLAIRE-EUGÉNIE( $\left.{ }^{*}\right)$}

I. Le Puits à Muire est réuni à la Sannerie. - La réforme de l'administration des salines de Salins et leur amodiation (I6OI). Visite des bois affectés à la cuite des muires (I602).

Ni les Articles rédigés par Jehan de Pomereulx en 1542 , ni l'Ordonnance du 26 janvier I $565 / 6$ qui en avait repris les dispositions n'ávaient eu tous les bons effets qu'on en pouvait attendre en raison de l'incurie et de la diversité des Justices chargées de leur application. Ia confiscation des biens de Guillaume d'Orange en I 568 n'avait apporté aucune amélioration à l'état de choses antérieur: les Procureurs de Chalon (I) en avaient conservé l'administration pendant la confiscation et lorsque Philippe II les réunit au Domaine en I 593 (2), la juridiction des bois resta délaissée aux juges qui l'exerçaient auparavant.

Les dispositions prises par les Souverains pour conserver les bois des environs de Salins, en régler l'exploitation et en réservei les produits aux salines ne pouvaient d'ailleurs empêcher leur Saunerie d'avoir à souffrir de la concurrence, si étroitement réglementée fût-elle, du Puits à muire. Le rachat de cet établissement pouvait

(*) Voir Rcv. For. Franc., avril 1951, p. 292 et février 1953, p. 94.

(I) La délimitation de la forêt du Jura, effectuée par les Procureurs de Chalon pendant la confiscation, désavouée en r606 par les Réformateurs, donne la mesure de leur fidélité (Cf. P.V. de visite de 1606: Résolutions prises. Arch. Doubs, Ch. des Comptes, B 2III).

(2) Cf. Arch. Doubs, Ch. des Comptes. Saunerie. Inventaire (d'après Max Prinet. L'industrie du sel en Franche-Comté avant la conquête française). Les comptes des bois de Chalon ont été tenus jusqu'en I594 (Arch. Doubs. Ch. des Comptes, B. 2109). 
seul y mettre un terme tout en assurant à la Saunerie la disposition de muires bien plus riches (3).

Au $\mathrm{Xv}^{\circ}$ siècle, Charles le Téméraire avait déjà fait racheter les quartiers du Puits à muire (4) qui s'étaient alors trouvés à vendre. En I57 I, le duc d'Albe entreprit de les racheter tous. A la fin du $\mathrm{XVI}^{\mathrm{\theta}}$ siècle, la plupart avait été cédés au Souverain. Seuls, quelques Rentiers ecclésiastiques, sous le couvert du droit canon, refusaient encore de vendre les leurs. Les Souverains (5) s'en portèrent amodiataires et le Puits à muire fut réuni à la Saunerie.

A cette occasion, des modifications profondes furent apportées au régime d'exploitation de la Saunerie. L'administration que Philippe le Hardi y avait établie fut supprimée. Les salines de Salins furent amodiées à un fermier qui les exploita sous le contrôle d'un Juge nommé par le Souverain. Le premier bail, dont Pierre Faulche, seigneur de Nancray, fut le bénéficiaire est daté du 28 septembre I60I (6).

Le fermier rétait maintenı dans la jouissance de tous les bois du Domaine, des Communes et des Particuliers antérieurement affectés à la cuite des muires aux conditions fixées par les Souverains. Ceuxci se réservaient avec les droits de Justice le pouvoir d'en régler et surveiller l'exploitation. A l'entrée en jouissance du fermier. puis d'année en année. il devait être procédé à la visite contradictoire cle tous les bois affectés à la cuite cles muires pour en noter l'âge et l'état. Le fermier recevait d'ailleurs à son entrée et devait laisser à sa sortie provision de bois suffisante pour assurer la marche ininterrompue des salines.

La visite contradictoire des bois prescrite par l'article 10 du bail d11 28 septembre I6or fut faite au cours des mois de février. mars et avril i602. Jean Stercue et Jean Denetuleres, conseillers à la Chambre des comptes de Lille y avaient d'abord commis pour les Souverains deux officiers de l'ancienne administration de la Saunerie, Pierre du PRrL, clerc des rôles et Jean Boutechou, clerc ventier, assisté d'Etienne Petit-Viennet et d'Etienne Amiel, fasseurs. Mais peu après le début de l'opération, sur une nouvelle commission, Simon Musy qui venait d'être institué juge des sauneries, se joignit à eux et prit la direction des travaux. Le procès-verbal qui en fut rédigé par ses soins apporte sur l'état des bois affectés à la cuite des

(3) Aussitôt après la réunion des deux salines, un cạnal fut aménagé pour amener à la Saunerie les muires du Puits à muire.

(4) La propriété du Puits à muire était divisée en quartiers dont les détenteurs étaient appelés Rentiers.

(5) L'Archiduc Albert et l'Infante Isabel Clara Eugenia.

(6) Cf. Max Prinet. L'Industrie du sel en Franche-Comté avant la conquête française. Besançon, I900. Cet auteur qui met en parallèle la prospérité des salines au temps des fermiers et les difficultés rencontrées dans l'exploitation de la Saunerie au $X^{\prime} I^{\ominus}$ siècle, paraît avoir méconnu à quel point la réunion du Puits à muire à la Saunerie en avait modifié les conditions d'exploitation. 
muires au début du $\mathrm{xVJI} \mathrm{I}^{\mathrm{e}}$ siècle un témoignage d'une précision rarement atteinte à cette époque (7).

Le traitement des taillis avait évolué depuis I542. L'exploitation cles taillis en tenant laie et sans en rien délaisser, ne s'était imposée ni dans les bois oì les exploitations usagères maintenaient le plus grand désordre, ni même dans toutes les forêts banales. L'usage s'était en effet établi de donner certaines de ces forêts " en nombre ». Le fasseur était libre d'y exploiter à son gré pendant une période déterminée, à charge de fournir à la Saunerie une quantité de bois fixée à l'avance. Le plus souvent, il exploitait en champelant (8). Cependant, au moins dans les forêts de médiocre dimension, il ne dispersait pas chaque année la récolte sur toute l'étendue de la forêt, mais la réalisait au cours de passages successifs à plusieurs années d'intervalle. C'était là le mode d'exploitation qu'on a appelé depuis avec plus ou moins de bonheur le traitement en taillis fureté. Enfin, la rotation des coupes avait été très sensiblement allongée et portée aux environs de 9 ans.

Les taillis établis sur l'emplacement de sapinières ruinées au $\mathrm{xv}^{\ominus}$ siècle par les abus de la jouissance usagère (La Joux, Maubelin, Loyaubault, Arc) étaient restés en fort mauvais état. Pillés et abroutis, ce n'était plus, du moins sur les sols rochet1x, qu'épines et buissons. Cependant, si ces bois n'étaient pas retournés dans leur " bonne et primitive nature ), les dispositions des Articles de $\mathrm{I}_{542}$ et de l'Ordonnance de $1565 / 6$ n'y étaient pas restées rigoureusement sans effet. La présence du sapin s'y était maintenue, mais il n'y était représenté que par de jeunes sujets, et, si quelqu'un était délaissé jusqu'à la dimension d'un chevron, c'était communément parce qu'il était " raffaux et noueux ) (9).

Dans la description des chênaies et des sapinières, Simon Musy a distingué les arbres en quatre ( espèces ) (classes de grosseur) suivant les produits qu'on en pouvait tirer (pièces de dimensions supérieures à celles d'une panne pour la première, panne pour la deuxième, chevrons pour la troisième, demi-chevrons et lattes pour la quatrième) et indiqué pour les arbres de chaque catégorie la distance moyenne qui les séparait dans le peuplement ( compensant les plus éloignés avec d'autres qui sont plus proches 》). Ces descriptions mettent en évidence le renouvellement à peu près complet du matériel cle ces forêts depuis I 542 .

(7) Cf. Arch. Doubs. Chambre des Comptes, B. 2rio.

(8) « Champeler est couper le gros bois et laisser le petit 》 (Simon Musv).

(9) Les effets de cette rigoureuse sélection à rebours dénoncée par Jehan de Pomereulx en 1542, comme par Simon Musy en 1602, sont encore sensibles dans les bois où elle s'est exercée pendant plusieurs générations du $\mathrm{xv}^{\mathbf{e}}$ au $\mathrm{XVII}^{*}$ siècle, et singulièrement dans la forêt de la Joux, la plus éloignée de massifs tels que le Jura qui ont bénéficié à peu près sans interruption de la sélection naturelle. 
Au bois Mouchard (10) divisé en quatre quartiers, les tröis derniers subdivisés eux-mêmes en trois ou quatre parts, les chênes da la $\mathrm{I}^{\text {re }}$ espèce, fort rares dans les deux premiers quartiers et dans une part du troisième, étaient distants de 6 toises dans le surplus du troisième et de $4 \mathrm{I} / 2$ dans le quatrième. Ceux des trois autres espèces, " confondus et mélangés par ensemble ", étaient distants d'I, I I/2, 2 et 3 toises suivant les parts (II). En fait, ce n'esț qu'à l'Est de la forêt, dans les premiers quartiers qu'on trouvait encore de rares témoins de la chênaie médiévale, d'ailleurs " escoulonnés secs en cime ) (I2). Les chênes de la $I^{\text {re }}$ classe, assez nombreux à l'ouest de la forêt dans les troisième et quatrième quartiers n'étaient vraisemblablement que les sujets les plus avancés de l'ahondante renaissance décrite par Jehan de Pomereulx. Il s'agit là en effet de cette partic du bois Mouchard, la plus proche d'Arbois que les déprédations des habitants de cette ville avait très largement ouverte à une date antérieure à I 542. Bien que n'offrant que des différences d'âge peu marquées entre ses diverses parts et, communément, dans chaque part, entre ses divers éléments, la chênaie du bois Mouchard était peu régulière. Néanmoins, son couvert était partout assez complet pour faire obstacle à la croissance des menus-bois.

La fassure n'avait été établie que depuis peu d'années dans la Fresse de Vers et de Montrivel (I3). Le fasseur y pouvait exploiter avec les bois d'essences diverses les chênes, en en clélaissant les plus: beaux de toise en toise. En I602, la forêt était peuplée de plançons de chêne et de menus bois. Bien que réputée bois cle haute futaie (I4) on n'y trouvait que de jeunes bois, les plus gros propres à faire chevrons, et par places, singulièrement au nord dı massif et sur les lisières, si clairs que les commis du fermier purent cl'abord prétenrre que ce n'était qu'un bois taillis. I.es chênes y étaient distants én moyenne de 4 à 5 toises clans le $I^{\text {er }}$ quartier au, nord, clc 2 à 3 toises

(10) Cf. P. V. de visite de 1602 , pages $79 \mathrm{R}$ à $83 \mathrm{~V}$.

(II) Le nombre des arbres à l'hectare pourrait atteindre 3000 s'ils étaient régulièrement disposés à I toise d'intervalle. Mais il s'agit là d'arbres irrégulièrement disposés et tout indique (notamment les dispositions réglementaires dont il sera parlé ci-après) que les distances mesurées étaient celles d'un arbre à l'arbre de même espèce la plus proche. Le nombre à l'hectare des arbres distants d'une toise était donc très inférieur à 2500 ou 3000 .

(I2) Escoulonné se dit, soit d'un arbre dont la cime a été rompue ou conpée, soit, et c'est ici le cas, d'un arbre dont la cime est sèche.

(13) $\mathrm{P}$. V. de visite de 1602 , pages $30 \mathrm{~V}$ à $36 \mathrm{R}$.

La forêt est aujourd'hui connue sous le nom de forêt domaniale de La Fresse. Avant la confiscation, les Officiers de Chalon s'étaient opposés à ce qu'on y établît la fassure « parce qu'elle était merveilleusement duisante à la maison de Vers à raison de la chasse »( $\operatorname{Ord}^{\mathrm{e}}$ du 26 janvier $1565 / 6$. Art. 102).

(I4) Comme l'établissait l'usage d'y amodier la glandée. La propriété de la glandée y était alors séparée de celle des bois. Elle avait été restituée en I6or avec les autres biens de la maison de Chalon à Philippe-Guillaume d'Orange. La propriété des bois, affectés aux Salines avait été conservée aux Souverains. 
dans le $2^{\circ}$, de 2 toises dans le $3^{\circ}$ et le $4^{\circ}$, de 2 à 3 toises dans le $5^{\circ}$ au sud, mais fort irrégulièrement répartis: à $1 / 2$ toise l'un de l'autre, là où le peuplement était le plus épais, à Io ou I 2 là où il était le plus clélabré. On y remarquait en outre (quelques vides rocheux ou gazonnés. Le clélabrement d'une partic importante de la forêt fut imputé, sur quelques points, à l'ingratitude du sol, et plus généralement aux abus non réprimés des riverains qui, sous couvert d'un droit d'usage aux morts-bois et au pâturage, coupaient les chênes là oì ils étaient à leur main.

Les Grands bois de Vers (15) n'avaient pas été moins maltraités. Pour un quart, le plus proche de Vers, ils étaient réduits en buissons. Sur les trois autres quarts, sauf les vides dus aux abus de dépaissance, ils étaient peuplés tant de vieux chênes que de jeunes plançons de la mème essence, mêlés d'autres bois, les vieux chênes fort rares, distants de 20 à 30 toises " noueux, demi-secs et pelı branchus, ayant été plusieurs d'iceux escoulonnés et coupés par dessus $)$. les plançons distants en moyenne de 2 toises, propres à faire des pièces de 2 toises de long, quelques-uns cependant de 4 à 5 toises.

Les vieux bois qui occupaient une place prépondérante dans la chênaie médiévale avaient à peu près disparu de ces forêts dévastées au cours du siècle précéclent. Nais à la faveur du mode d'exploitation en usage. et malgré les abus de toutes sortes, la chênaie s'y était très généralement reconstituée avec une extrême facilité, même aux Grands bois de Vers et à la Fresse sous un climat déjà fort rigroureux. Bien que sensiblement équiennes, les peuplements étaient peu réguliers et les arbres qui les formaient généralement courts et trapus. Aux termes mêmes du procès-verbal de visite de r6o2, ne levait-on pas choisir puur les épargner " les plus propres à porter paisson )?

I_es données numériques constituent un des éléments les plus précieux, bien que d'interprétation délicate, du témoignage porté par Simon Musy sur l'état et la consistance des peuplements des sapinières. Elles ont été consignées ci-après dans un tableau où, pour permettre la lecture directe de la richesse relative de chaque peuplement en arbre des espèces distinguées, on a substitué aux distances d indiquées au procès-verbal les quotients $\frac{\mathrm{IOOO}}{\mathrm{d}^{2}}$ qui mesurent cette richesse dans un système où elle est évaluée conventionnellement ì IOOO pour $\mathrm{d}=\mathrm{r}$ toise.

TABLEAU,

(15) $\mathrm{P}$. V. de visite de 1602 , pages $36 \mathrm{~V}$ à $38 \mathrm{~V}$. 


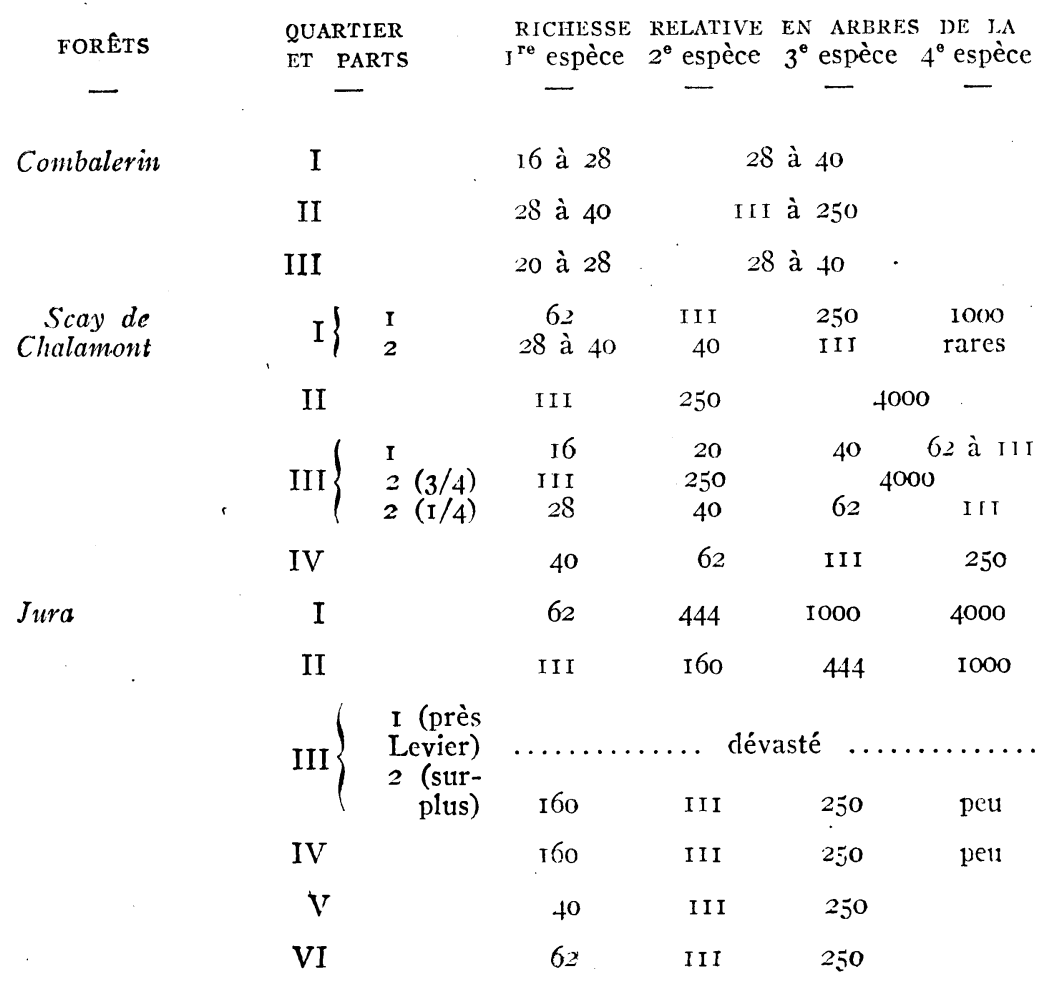

Ces chiffres n'ont pas la précision qu'on peut atteindre aujourd'hui zrâce aux comptages. Ils donnent néanmoins sur la consistance des peuplements des indications valables, corroborées par l'ensemble des faits rapportés par Simon Musy ou établis par la conférence de son témoignage et de celui de Jchan de Pomereulx. I a présence aux visites de deux Officiers de la Saunerie, des commis du fermier, des préposés à la surveillance et à l'exploitation des bois, tous diversement intéressés à l'exactitude de l'état des lieux ainsi dressé et fort prompts, le cas échéant, a en relever les erreurs, constitue d'ailleurs une sérieuse garantie de cette cxactitude.

I,a forêt de Combalerin (I6) avait gravement souffert au cours des 20 dernières années d'ahus d'exploitation, moins toutefois au ceitre (Q II) qu'au sud (Q III) et surtout qu'au nord ( $($ I). En I 542, Jehan de Pomereulx l'avait trouvée dévastée sur la nıoitié de sa contenance. En I602. Simon Musy la décrit pillée sur toute son

(16) Cf. P. V. de visite de I602, p. $38 \mathrm{~V}$ à 44 R. La forêt de Combalerin, d'une contenance de I50 ha environ était située dans la combe de ce nom. Entièrement enclavée dans le bois de la Joux de Salins, elle lui a été réunie pour former la forêt domaniale de la Joux 
étendue. Le fasseur ct le forestier imputèrent ces dégradations aux exploitations faites pour la Sauneric ou concédées à des particuliers par la Chambre des Comptes, les Officiers de la Saunerie, non sans apparente ra:son, à la multitude des délits. La forêt de Combalerin y était en effet singulièrement exposée tant en raison de sa situation que de son étendue médiocre. Quoi qu'il en fut, c'était là une forêt furetée, dans le sens péjoratif que lui attribuaient les forestiers français de l'Ancien Régime.

La forêt de Scay de Chalamont (I7) allongée sur les deux versants d'un chaînon, l'un assez rétendu vers le N.-O., l'autre fort court vers le S.-E. était également fort exposée aux délits par sa configuration. Elle avait aussi à supporter les abus de plusieurs usagers ou prétendus tels et singulièrement du $\mathrm{R}^{\mathrm{d}}$ Abbé de Mont-SainteMarie et Rozières et de ses grangers de Crissay, Gouailles et Montorge. Après l'orvale qui en avait dévasté la plupart en I527, les peuplements de la forêt s'étaient reconstitués. La fassure établie après $\mathrm{I}^{542} \mathrm{y}$ avait aidé en dégageant les sapineaux des morts-bois. Au nord du chemin de la Galande, le peuplement assez clair sur le versant N.-O. (Q I. 2) et délabré sur le versant S.-E. (Q I. I) où les grangers de Crissay venaient couper le bois « non seulement pour le bâtiment... mais encore pour le chauffage et la clôture de leurs héritages ") comprenait toujours quelques très vieux bois (I8). Plus au sud et jusqu'au chemin de la Tour (Q II) le peuplement bien conservé, car le quartier était de " traite difficile », était encore jeune; régulier et très dense. Au sud du chemin de la Tour, le peuplement du versant S.-E. était analogue à celui du $2^{\text {ei }}$ quartier, bien plus clair cependant au voisinage de la crête (Q III. 2). Sur le versant N.-O., jusqu'à la vie de la Tille (Q III. I), Simon Musy le décrit semé d'arbres chablis, rompus et secs. Sur cette partie de la forêt d'accès facile et que l'orvale de ${ }^{527}$ avait sans doute plus ou moins épargnée, les exploitations faites pour la Saunerie et, sur mandement de la Chambre des Comptes, pour les particuliers, jointes aux dégradations du $\mathrm{R}^{\mathrm{d}}$ Abbé dont il avait cause pendante en la Justice de Chalamont et de ses grangers de Gouailles et de Montorge, avait abusivement ouvert le peuplement qui s'effondrait. Enfin au sud de la vie de la Tille (Q IV) le peuplement était inégal, mais en moyenne fort clair. Sans doute, ce quartier limitrophe des bois de la Joux de Salin et de Loyaubault avait-il eu à souffrir, non seulement des abus des grangers de Montorge, mais aussi des incursions des usagers des bois voisins.

(I7) Cf. P. V. de visite de I602, pages $48 \mathrm{R}$ à $58 \mathrm{~V}$. La forêt de Scay de Chalamont, d'une contenance de plus de 400 ha, réunie à la partie de Maubelin restée domaniale, forme aujourd'hui les $6^{\circ}$ et $7^{\circ}$ série de la forêt domaniale de Levier.

(I8) Quartier désigné en I6I I pour le recrutement des bois nécessaires à la fabrication des bosses (voir infra). 
La forêt du Jura (I9) était la plus ample et la plus belle des sapinières affectées aux salines. Des vieux peuplements en voie de dislocation dès $\mathrm{I} 542$, il ne restait que peu de témoins, plus nombreux peut-être au N.-E. du massif à l'est du sentier de Levier à Labergement (20) où ils survivaient épars (2I) au milieu d'une renaissance fort épaisse bien que malmenée sur les lisières par les habitants de Levier et de Labergement (Q I). A l'ouest du sentier de Labergement et jusqu'à la vie du Moulin, le peuplement, plus anciennement dégagé, était sensiblement moins dense, mais formé d'arbre plus gros (Q II). De la vie du Moulin aux Biefs, sur la moitié de la contenance du massif, il était constitué par une futaie complète de consistance moyenne " y ayant peu de rejetons et petits de la $4^{\mathrm{e}}$ espèce par le moyen que l'ombrage des gros empêche les petits de croitre ) (Q III 2 , et $Q$ IV). Les peuplements du $3^{\mathrm{e}}$ quartier à l'est du sentier de Salins (22) ne différaient de celui du $4^{\mathrm{e}}$ qu'au voisinage de Levier, des lisières au pré Henri Gaume, proche de la Louvatière, où les riverains l'avaient dévasté (Q III I). Au sud des Piefs se trouvait la partie du massif désignée auparavant sous le nom de forêt des Amourtoux. Le peuplement y était plus clair et les arbres moins gros. Sans doute faut-il l'imputer avec Simon Musy à l'activité des délinquants plus marquée à l'ouest du sentier de Salins, vers Villeneuve $(Q \mathrm{~V})$ qu'à l'est $(Q \mathrm{VI})$. D'ailleurs, si clans leur' ensemble, les peuplements du Jura paraissent moins éprouvés que ceux du Scay et surtout de Combalerin par le pillage des riverains, cc n'est qu'en raison de l'étendue de la forêt. Simon Musy y avait trouvé peut-être plus qu'ailleurs un prodigieux gaspillage de bois, mais, sauf les déprédations commises par les populations réfugiées au cœur des massifs pendant les guerres de I 595 et lors du passage cles troupes espagnoles en I60I, l'activité des délinquants intéressait surtout les cantons les plus faciles d'accès. Par négligence ou par esprit d'économie, le nombre des forestiers chargés de garder le Jura avait été réduit à deux: l'un résidant au siège de la Justice à Montmahoux était trop éloigné pour y pouvoir servir, l'autre « n'osait beaucoup fréquenter par les dits bois, par crainte d'être battı ou outragé »).

(19) Cf. P.V. de visite de I602, pages $60 \mathrm{R}$ à $67 \mathrm{~V}$. La forêt du Jura (avec la forêt limitrophe des Amourtoux qu'on y a réuni) avait une contenance de plus de 900 ha. Elle forme aujourd'hui les $\mathrm{I}^{\mathbf{r e}}, 2^{\boldsymbol{\theta}}, 3^{\boldsymbol{\theta}}$ séries de la forêt domaniale de Levier.

(20) Le sentier de Levier à Labergement était situé à l'ouest de la routc actuelle.

(2I) Quartier désigné en I6II pour le recrutement des bois destinés à la fabrication des bosses. (Voir infra).

(22) Le sentier de Salins reliait à l'ouest du Pré au Maire la vie du Moulin à la Grand'vie appelée improprement aujourd'hui la Voie romaine. 
2. La Réformation des bois affectés à la cuite des muires cst prescrite ( 1607$)$ et le Conseiller Guillaume de Steenlutys chargé d'y prósider (1605). Travaux des Commissaires.

I.e procès-verbal de la seconde visite des bois affectés aux Salines, commencée le 9 septembre I603, ne parait pas avoir été conservé, mais il est visé dans les lettres patentes données à Gand le 22 octobre 1604 par l'Archicluc Albert et l'Infante Isabel Clara Eugenia pour mettre un terme aux « entreprises, désordres et usurpations qui v étaient dénoncés ». Le Procureur Général au Comté de Bourgogne, Luc de Saint-Mauris, le Juge Simon Musy, le taxeur de bois Claude Pouler et le greffier de la Justice des Salines Claude Perrier étaient commis et recevaient pleins pouvoirs à cet effet. Puis, comme ils tarclaient à se mettre à la besogne, le maitre des Requêtes Guillaume de Steeniruys, Conseiller au grand Conseil de Malines, fut chargé de prendre l'affaire en mains. Aux termes des instructions qui lui furent données le 5 août I605, il s'agissait essentiellement de procéder au cours d'une visite à la diélimitation des bois et au règlement des droits d'usage, puis de revoir et amender l'Ordonnance de $1565 / 6$ (23).

Auparavant, les usagers avaient été mis en demeure de déposer leurs titres au greffe clu Parlement de Dole (24) et les bois conservés à la Couronne lors de la restitution cles biens de la maison de Chalon à Philippe Guillaume d'Orange avaient été rattachés au domaine de la. Saunerie (25).

I.es Commissaires se réunirent à Salins le 5 avril $\mathbf{6} 606$ pour procéder à la visite des bois. Claude Poulet, malade, s'excusa et Luc de Sarnt-Mauris empêché par les obligations de sa charge ne prit que peu de part aux travaux. Par contre, Guillaume de Stefnhuys et Simon Musy y appelèrent avec Pierre du Prel, Henri Boutecirou et le fasseur Petit-Viennet, " ci devant employés à la visite des bois ") (26) l'ancien procureur de la Saunerie Guyon Amel.

I a description des peuplements tient peu de place dans le procèsverbal de la visite de 1606 . Sans doute jugea-t-on inutile d'y revenir sur un travail déjà fait avec soin. Mais on y peut lire avec inté-

(23) Cf. Arch. Doubs Chambre des Comptes. B 2III. "Visitte générale des bois destinés à la cuitte des muyres des Saulneries de Salins, faite par Monsieur Desteenhuis du Grand Conseil de leurs AA.SS., Messieurs les Pro* cureur Général de SAint-Mauris et Juge Musy en l'an I606 》(Copie établic par le greffier de la Justice des Sauneries Vimet après la guerre de io ans, vers 1650 ).

(24) Cf. Arch. Doubs. Chambre des Comptes B. 2107. Lettres patentes du 3I décembre i6or.

(25) Cf. Arch. Doubs. Chambre des Comptes B. 242. Lettres patentes du 16 mars 1604.

(26) C'était, sauf Jean BouTEchou déjà remplacé par Henri lors des précédentes visites, l'équipe d'anciens officiers de la Satunerie qui avait assisté Simon Musy dans la visite de I602. 
rêt le recolement détaillé des bois coupés depuis la visite de 1605 . Au bois Mouchard par exemple, on dénombra pour $7^{2}$ chênes marqués 394 chênes abattus sans marque et au Jura pour I69. sapins marqués 87i sapins abattus sans marque. Encore doit-on admettre que si les commissaires purent se faire montrer par les fasseurs tous les bois marqués, le dénombrement des bois abattus sans marque resta nécessairement très incomplet.

La description des limites et les enquêtes relatives aux droits prétendus par les riverains sur tout ou partie des massifs tiennent une place importante dans le procès-verbal de visite de I606. L'appropriation des bois communs où les habitants d'une setule communauté exerçaient des croits d'usage était très généralement un fait acquis à la fin du $\mathrm{XVI}^{\theta}$ siècle. Celle des massifs oì ces droits étaient cxercés indivisément par plusieurs communautés avait été plus malaisée. L'entreprise de chaque communauté était nécessairement limitée à la partie du massif voisine du village, la plus fréquentée par les habitants (27). Encore fallait-il que ceux-ci soient assez forts pour faire respecter par les atitres usagers les quartiers qu'ils entendaient se réserver et mettaient en ban à cet effet (28). Ces quartiers furent compris dans les bois des souverains, mais, semble-t-il, sans que leur mise en ban fut levée pour les usagers. A l'occasion cependant, les commissaires recommandèrent qu'on veillât à empêcher les communautés que les avaient réservés de se les approprier. Ils sont devenus néanmoins pour la plupart propriété communale (29).

Non seulement le peuplement des quartiers mis en ban était en bien meilleur état que celui du surplus des massifs. mais encore plusieurs communautés avaient entrepris d'en modifier la nature. Les habitants de I.cvier avaient reconstitué quatre sapinières au nord le Maubelin et ceux de Lemuy une chènaic au nord de la Joux. A cette fin, ils avaient appliqué les procédés éprouvés de la sylviculture médiévale, laissant croître sapineaux ou chêneaux et " décombrant tous autres menus bois afin de leur donner plus belle croissance ") (30).

(27) Cf. P. V. de visite de I606. Visite de la Joux de Salins « ćtant pour ce les limites bien incertaines, d'autant que plusieurs tâchent de s'en approprier quelque portion au plus près de soi... » « Ils (les habitants de Nan) ont dit qu'ils y avaient tel et semblable droit qu'au surplus de la Joux et qu'ils l'avaient épargné et mis en ban parce qu'il était proche d'eux, n'entendant point qu'autres quelconques y eussent usage... $\gg$

(28) Cf. P. V. de visite de I606. Visite du bois d'Arc. Témoignage des habitants de Cernans au sujet des quartiers mis en ban par ceux de Villers « ont dit de plus que lesdits de Villers prétendaient déjà de sa propriété sous environ 30 ans, et que pour y obvier, les habitants de Cernans y allaient en nombre de 20 à 25, mais lesdits de Villers survinrent deux fois autant qui les chassèrent et prirent tout le bois qu'ils avaient coupé sauf trois ou quatre chariots qu'ils amenèrent... ».

(29) Les Essarts lointains, La Vignière, La Lappe (Arc), Le bois Tallard (Maubelin), etc...

(30) Cf. P. V. de visite de 1606 . Visites de Maubelin et de la Joux. 
Les commissaires n'avaient pas trouvé les forêts banales mieux défendues des entreprises des riverains que les hois communns. Ils clurent rejeter les prétentions injustifiées de l'Abbé de MIONT-SAINTrMARIE sur un quartier de la forêt de Scay, lésavouer le bornage suspect de la forêt du Jura conscnti par les Procureurs de Chalon pendant la confiscation et poursuivre devant le Parlement de Dole les halitants de Gevresin qui avaient espéré s'arrondir davantage cil posant de fausses hornes (3I).

La délimitation des bois et forêts ne fut pas suivie de bornages. Comme en droit comtois " le bois acquiert le plain » (32), de tclles opérations pouvaient paraitre désavantageuses au propriétaire du bois. Les commissaires cependant avaient remarqué que les habitants de Levier brûlaient systématiquement les arbres de lisière pour agrandir leurs communaux aux dépens de la forêt du Jura (3.3).

Après la visite des bois, les comnissaires prirent des résolutions sur les difficultés qui s'y étaient présentées (34) et arrêtèrent en date du 15 décembre r6o6 le rôle des usagers. Puis il s'attachèrent à revoir avec le concours des officiers de l'ancienne administration quii les avaient cléjà assisté dans la visite, outre l'Ordonnance de I $565 / 6$, toutes les dispositions intéressant la Juridiction des Sauneries. La révision des règlements particuliers des bois (les articles particuliers de $\mathrm{I}_{542}$ et I9 et sq. de l'Ordonnance de I $_{565 / 6)}$ fut cependant différée et laissée aux Officiers des Sauneries. Ces travalix aboutirent à la rédaction des trois Ordonnances qui furent données par l'Archiduc Albert et l'Infante Isabel Clara Engenia les 27 août I607. 22 septembre $\mathrm{s} 607$ et 8 juin I6II.

3. Les ()rdonnances de l'Archiduc Albert it de l'Infante Lsabelle Claire İugénie.

\section{L'Ordonnance du 22 septembre 1007}

Avant l'étude des Ordonnances des 27 août r607 et 8 juin I6I I relatives à l'exploitation des bois, il convient d'analyser sommairement l'Ordonnance du 22 septembre 1607 (35) qui devait en assurer la conservation par le règlement des droits d'usage et la répression efficace des mesus.

La Juridiction des Sauneries était étendue aux bois du partage de Chalon réunis au domaine de la Saunerie en $160+$ et à tous les bois quelqu'en fût le propriétaire, destinés à la cuite des muires

(3I) Cf. P. V. de visite de I606, les visites du Scay et du Jura et in fine, les résolutions prises.

(32) Les accrues appartiennent au proprićtaire du bois.

(33) Cf. P. V. de visite de 1606. Visite du Jura.

(34) Cf. P. V. de visite de I606. In fine.

(35) Cf. Bibl. M. Salins M. $n^{\circ}$ I28. Ordonnances des Souverains de 12 Franche-Comté, relatives aux Sauneries de Salins. 
dans le ressort du Parlement de Dole. (Art. 4 à II). La charge de pourvoir à l'entretien des ponts et chemins nécessaires à la conduite des bois et des sels (Art. I9) et le pouvoir de contraindre en cas de nécessité charretiers et paysans de faire 3 jours par semaine de charroi pour la Saunerie, et ce, nonobstant peste et contagion (Art. 2I à 24) restaient en outre dévolus au Juge des Sauneries.

Le Juge ne pouvait amoindrir les peines portées par les Ordonnances (Art. I). Les procureurs et avocats attachés à son siège devaient veiller aux poursuites (Art. 2 et 20). Ses jugements étaient exécutoires nonobstant appel jusqu'à roo livres d'amende. L'appelant passible de peines corporelles restait détenu. Le contumace devait se rendre priscnnier. Faute d'acquitter l'amende dans un délai (le + mois à la caisse du receveur des Exploits, le mésusant pouvait être saisi de corps (Art. I2 à I8).

Les défrichements étaient prohibés jusqu'à trois lieues de Salins et ce, sauf récompense, nonobstant titres contraires (Art. 26 à 28). L'établissement de forges était interdit juscu'à 4 lieues de Salins (Art. 32 et 33), la fabrication, du salpêtre jusqu'à 6 lieues (Art. 34). Celle du charbon était interdite clans les bois affectés à la cuite des muires, et jusqu'à 3 lieues de Salins dans les bois communaux si ce n'est du consentement des prud'hommes (Art. 29 à 32). Aucune scierie nouvelle ne pouvait être établie jusqu'à 4 lieues de Salins et 2 lienes des forêts banales; les scieries anciennes étaient assujetties à l'observation de règlements établis par le Juge et ne pouvaient recevoir de plots qui n'aient été préalablement marqués (Art. 35 à 37 ).

Les chênes, chêneaux, sapins et sapineaux coupés en délit pouvaient être suivis jusqu'à 6 lienes de Salins. Les détenteurs de plots de ces essences étaient tenus l'en établir l'origine. Toute personne avait le droit d'arrêter le délinquant, nul ne pouvait s'y opposer. (Art. 38 à 44).

J'usager ne pouvait prenclre dans une forêt banale nul chène ou sapin en estant, mème mort, qu'il n'ait été cl'abord marqué et délivré. L'usager au bois mort et aux morts-bois ne pouvait enlever chêne ou sapin gisant qui n'ait quitté l'écorce. La clélivrance cles chênes et sapins, jeunes et vieux, pour l'usage du feu, barre ou cloison d'héritages était prohibée (Art. 45 à 49). Les bois délivrés clevaient être équarris sur place et enlevés dans le délai d'un mois (Art. 50 et 5I). Dans les hois de la Joux, Maubelin. I oyaubault it Arc, la coupe des sapins et sapineaux était interdite à tous. usagers et fasseurs, pour la clurće de 20 ans (Art. 52). I,écorçage était prohibé et tout détenteur d'écorces était tenu d'en déclarer la provenance (Art. 54 et 55 ).

I.c pâturage était interclit dans les coupes jusqu'à la troisième feuille (Art. 56). I.es communautés ne pouvaient vendre leurs bois ct jâtures sans un congé dı Parlement. (Cette pratique lont elles 
usaient pour éteindre leurs dettes aggravait les usages supportés par Jes bois du Souverain) (Art. 66). Elles étaient tenues de désigner rles gardes pour les bois dont elles avaient l'usage. Ceux-ci recevaient pour salaire le quart des amendes prononcées sur leurs dénonciations (Art. 62).

Il était interdit de mettre le feu aux champs, pâtures ou huissons proches des bois, jusqu'à 6 lieues de Salins, et aux bergers (1. faire du feu sous les chênes et les sapins (Art. 68).

Il était interdit aux Officiers de la Saunerie d'acheter des coupes de bois taillis jusqu'à 6 lieues de Salins (Art. $6_{3}$ à $6_{5}$ ).

I.es infractions aux dispositions de l'Ordonnance étaient généralement punies d'amendes proportionnelles à l'importance dù délit. Flles étaient fixées, par exemple, à 300 livres pour la construction d'une forge à distance prohibée, à Io livres par toise carrée de futaie et ro livres par arbre et à Ioo sous par toise carrée de taillis pour les défrichements, à Io livres par pied et 20 sous par fassin et farclelet (36) pour la coupe de chêne ou sapin, à 25 livres par troupeau et 60 sous par bête pour pâturage dans les jeunes revenues, etc... A ces amendes s'ajoutaient la démolition de la forge. le paiement d'intérêts en cas de défrichement, la confiscation du bois, des chariots et des attelages, s'il s'agissait d'un délit de bois. Celui qui s'était opposé à l'arrestation d'un délinquant de bois était puni d'une amende égale à quatre fois la valeur du bois, des chariots et des attelages. L'achat par un officier de la Saunerie d'une coupe à distance prohibée était puni de la confiscation de la coupe et du prix.

L'amende était doublée pour les délits commis la nuit (Art. 75). Le récidiviste, puni d'une amende double la deuxième fois et triple la troisième fois, était banni des bois pour trois ans la deuxième fois et à vie la troisième. Si une communauté avait mésusé en corps, clle pouvait être alors privée de son droit d'usage (Art. 74).

L'amende pour délit de pâturage frappait le propriétaire dı̀ bétail, mais celui-ci pouvait exercer un recours contre le pâtre. Les communautés étaient responsables des pâtres qu'elles avaient nommés. Les maitres étaient responsables des domestiques et punissables avec eux si le délit avait été commis par leur ordre (Art. 57 à 6r).

Des peines corporelles, pilori et fouet étaient substituées aux amendes si le délinquant ne les pouvait payer (Art. 76). S'il se clérobait à ces peines, il était banni des bois pour 5 ans (Art. 77).

Les forestiers convaincus d'avoir clétourné ou laissé clétourner. des chênes ou sapins étaient révoqués et punis du pilori, carcan et fouet. Si on ne les pouvait appréhender, ils étaient bannis des bois pour Io ans à peine de hart (Art. 72). Par contre, ils étaient protégés dans l'exercice de leurs fonctions. Les rosser pouvait valoir

(36) Fardelet est synonyme de chevasse (sorte de fagot). 
le hart ( $\Lambda$ rt. 83). Les dénonciateurs étaient récompensés par l'allocation du quart de l'amende; leurs noms étaient tenus secrets (Art. 8 I et 82 ).

La substance des dispositions de l'Ordonnance du 22 septembre I607 n'était pas nouvelle les commissaires l'avait puisée dans la coutume et dans les ordonnances antérieures des Souverains (37). Ils n'avaient innové que pour rendre la répression plus efficacc.

\section{L'Ordonnance du 27 août I6o7}

L'Ordonnance du 27 août 1607 (38) est une révision des Articles généranx de 1542 , enrichie de deux titres d'importance très inégale concernant l'institution de deux charges et offices de Marqueur et Contrôleur des bois et les fonctions de forestier.

En I 542 et en $1_{565 / 6}$, toutes les futaies affectées à la cuite des muires étaient du partage de Chalon et la marque en appartenait aux Officiers de Chalon. Par Ordonnance en date du 23 janvier I593 (38), Philippe II l'avait confiée à un Gruyer et Contrôleur général des fassures dont la réunion des bois de Chalon au domaine de la Saunerie devait entraîner la suppression. Deux officiers furent donc institués à la Saunerie qui eurent pour charge dans leurs circonscriptions respectives (39) la marque des futaies et la surveillance générale des bois et chemins dépendant de sa juridiction (Art. I).

Ils étaient tenus de visiter les futaies tous les deux mois, les taillis tous les trois mois, de dresser des procès-verbaux de leurs visites et les transmettre au Juge, de dénoncer au Juge, au Procureur et aux Avocats les abus constatés, de veiller à l'entretien des chemins. Ils pouvaient arrêter les délinquants en cas de flagrant délit, visiter les domiciles des suspects, saisir les bois et les faire transporter. Forestiers, fasseurs, guettes et compteurs de bois (40) les devaient assister (Art. 2 à 7).

Chacun d'eux était pourvu de deux marques distinctes, l'une pour les bois destinés aux usagers, l'autre pour les bois destinés à la Saunerie (Art. 8).

Ils ne devaient marquer pour les usagers, sauf sentence du Juge, que les chênes ou sapins portés sur une liste après que les bénéficiaires en aient fait paraitre l'amploi et la nécessité, pour la Saune-

(37) Quel qu'en soit l'intérêt, l'étude des sources de l'Ordonnance du 22 septembre 1607 dépasse le cadre d'une étude dont l'objet est l'évolution de la sylviculture et non celle du droit pénal forestier.

(38) Cf. Bibl. M. de Salins. M. n ${ }^{\circ}$ I28. Ordonnances des Souverains de 1a Franche-Comté relatives aux Sauneries de Salins.

(39) Les bois de la limite d' $\Lambda$ val, la forêt du Jura et les bois d'Arc et de Maubelin d'une part, ies bois de la limite d'Amont (sauf le Jura, Arc et Maubelin) de l'autre.

(40) Les guettes et les compteurs de hois étaient de bas-officiers de la Sa1nerie. 
rie, que les chênes et sapins jugés nécessaires à la provision clu fasseur (4I), sans dépasser le nombre fixé par les Ordonnances et sans attoucher atix arbres situés à moins de deux toises des linites. Dans le choix des arbres à délivrer, ils devaient s'inspirer de règles qui seront analysées ci-après (42). Ils étaient tenus de marquer pour la fassure les bois gisants qui y étaient propres. Enfin, ils devaient tenir, jour par jour et par forêt, un registre des bois naiqués avec indication de l'emploi ou du rendement selon qu'ils étaient (lestinés aux usagers ou à la Saunerie et en adresser de six mois en six mois un extrait au Juge (Art. I I, I2, I4 à 23).

Ils devaient assister aux visites du Juge au cours desquelles il était procédé au recolement des bois marqués. I1s devaient sc contenter de leurs gages et frais de bouche duement taxés sans jamais recevoir d'argent des usagers. Leurs négligences étaient punirs d'amendes, leurs prévarications de peines corporelles sans préjudice des intérêts (Art. 25, I3, 9 et Io).

Les titres de l'Ordonnance consacrés aux fasseurs et charretiers (Art. 26 à 50 et 58 à 68) sont sensiblement plus développés que les articles de I542. On y peut relever quelques dispositions nouvelles.

Si l'exploitation en tenant laie restait prescrite aux fasseurs sauf dérogations portées dans les Ordonnances, ceux-ci." ne devront couper le bois menu qui ne pourrait être employé à l'usage de fassin et de fardelet ». Le traitement en taillis régulier était donc clélaissé pour le traitement en taillis fureté, cléjà introduit au siècle précédent dans mainte fassure tenue en nombre. Les dispositions tendant à obliger les usagers à exploiter dans la laie du fasseur qui s'étaient révélées inapplicables n'étaient d'ailleurs pas maintenues (Art. 3I et 32). Liabattage des- taillis était interdit pendant la montéc le la sève en avril, mai et août (Art. 37). Pour prévenir las usurpations, les fasseurs devaient abattre les taillis juscu'à l'extrềme limite aux confins d'un autre bois, mais levaient en réserver une toisc aux confins des héritages dont aucune horne ne les séparait. (Art. 47).

I.es fautes des fasseurs et des charretiers étaient punies d'amendes. Les fasseurs restaient chargés de veiller à leur fassure, même pendant le temps qu'ils n'y exploitaient pas. En cas de dissimulation ou de grande négligence, ils encouraient une peine double cle l'amende qui aurait frappé le délinquant, en cas de prévarication la destitution et une peine arbitraire (Art. 44). Les peines étaient doublées en cas de récidive (Art. 48). Les fasseurs pouvaient être destitués par le Juge, si celui-ci estimait qu'ils avaient manqué à leurs devoirs (Art. 49).

(4I) Les marqueurs ne devaient marquer les bois qu'au fur et à mesure des besoins du fasseur, de peur que celui-ci ne délaissât les queues et arbres difficiles à fendre.

(42) Voir infra, Sylviculture des Ordonnances. 
Quelques articles de l'Ordonnance (5 I à 57) sont consacrés aux revoirs des forestiers, à leurs salaires et aux indemnités auxquelles ils peuvent prétendre.

\section{L.'Ordomance du 8 juin $16 I I$}

L'()rdonnance du 8 juin I6I I (43), publiée à la justice des Saunerics le 7 nai I6I2 est la revision des articles particuliers de ${ }_{542}$ (Art. I9 et sq. de l'Ordonnance de $\mathrm{I}_{56} 6 / 6$ ).

Les bois destinés à la cuite des muires, où l'on remarque plusieurs bois et forèts qui n’y avaient pas encore été employés, entre autres la forêt de la Faye de Montrond et un quartier des bois de Chaux étaient divisés en 34 fassures (44') d'importance très inégale. Un règlement particulier était établi pour chaque fassure.

Le soin de régler l'exploitation des bois particuliers qui avaient appartenu aux Seigneurs du partage de Vignory et restaient à ce titre affectés à la cuite des muires était laissé aux propriétaires (45) tenus seulement d'en livrer tous les produits aux Sauneries.

L'Ordonnance dı 27 août I607 avait prescrit aux fasseurs de délaisser dans la laie qu'ils devaient tenir les bois qui n'étaient pas propres à faire fassin ou fardelet. Dans l'aménagement des taillis banaux où l'exploitation portait sur les bois de toutes essences sans cll épargnier aucune, il ne fut déroşé à cette règle que pour prescrire le recépage " tout a rez ) des recrues abrouties. Le règlement de chaque fassure indiquait les années de la prochaine exploitation (fui clevait recourir de 9 en 9 ans. L'exploitation courait pendant un. deux ou trois ans, suivant l'étenclue de la fassure et les ressurces du charroi, puis était suspendue jusqu'au terme de la rotation. Toutefois. dans les forêts de grande étenclue (la Fave de Valempouillères. la forêt de Chalon on de Fertans), on jugea expérlient d'instituer une fassure perpétuelle (où l'exploitation se poursuivrait sans interruption). A cet effet, les forêts furent divisées en trois quartiers à exploiter chacun au cours de périodes triennales succesives. La même disposition fut appliquée à la forêt de I.cutte, fort éloignée de Salins, par crainte de n'y pouroir disposer du charroi nécessaire si on l'exploitait d'un coup. Plusieurs fassures furent. sniviant l'usagre établi an ciècle précédent, laissées en nombrc aux fasseurs à charge d'en appliqucr cxactement le règlement.

I.es dispositions de l'article I7 des Articles de I542 prescrivant

(43) (f. Bibl. M. de Salins. M. nº r28. Ordonnances des Souverains de Franche-Comté relatives aux Sauneries de Salins et Arch. Doubs. Chambre les Comptes B $210 \%$.

(44) Le mot fassure désigne soit l'exploitation faite par le fasseur, soit le ou les bois dont l'exploitation et la garde étaient confiées à un fasseur.

(45) Sauf toutefois la fassure dite de Vignory qui fut aménagée comme un bois banal. Lors de la cession de leur part de la Saunerie au Souverain en 1.570 , les seigneurs de Vignory avaient laissé à la Saunerie le droit d'explositer ce bois moyennant une redevance de 2 engrognes pour roo fassins. 
aux usagers d'exploiter dans l'ordon du fasseur étaient restées lettre morte ct l'Ordonnance clu 27 aoht 1607 ne les avaient pas retenues. Les massifs de Moidon, de la Joux, de Loyaubault, (le Manbelin et d'Arc où de nombreuses communautés avaient leurs usages restaient livrés aux exploitations désordonnées des habitants qui y coupaient où bon leur semblait non seulement des bois pour leurs besoins, mais pour vendre à la Saunerie où ils étaient taxés comme bois communal. Ces errements, qui dataient de l'Ordonnance de Philippe Loyte (46) furent interdits (les communautés pouvaient en effet y trouver prétexte à s'approprier les bois) et pour engager les habitants à renoncer à la fabrication des bois destinés à la Sat1nerie, les règlements ramenèrent le prix de Ioo fassins fixé à 5 gros 2 engrognes (47) pour les bois faits par le fasseur, 4 gros pour le charroi et I4 engrognes pour la façon et la garde, à 4 gros et 8 engrognes pour les bois faits par l'habitant. Des exploitations conformes aux dispositions des art. $3 \mathrm{I}$ et 32 de l'Ordonnance du 27 août I6o7 ne pouvaient être pratiquées dans de tels bois. Les règlements prescrivait qu'on y fît courir la fassure de marteau en marteaú (48) aux endroits les mieux peuplés. Ceux des fassures de la Joux,Loyaubault, Maubelin et Arc rappelaient les dispositions de l'art. 52 de l'Ordonnance du 22 septembre 1607 , renouvelées des Articles de I 542, qui avaient interdit à tous, pour une durée de 20 ans l'exploitation de sapins dans ces forêts. Les forestiers étaient tout spécialement commis à leur garde.

Dans les futaies feuillues, l'Ordonnance du 8 juin I6I I avait à régler les deux sortes d'exploitation qui normalement s'y superposaient, celle des morts bois, conformément aux dispositions des articles 31 et 32 de l'Ordonnance du 27 août I6o7 et celle (les arbres clésignés par le marqueui suivant les prescriptions de l'article if de ladite Ordonnance.

On dut cependant tenir compte des droits d'usage aux morts-bois concédés aux riverains dans plusieurs massifs. C'est ainsi qu'à la Fresse de Vers et de Montrivel et au bois Mouchard, les règlements ne purent prescrire aucune exploitation assurée de morts-bois. Au bois de Chanx (49), le fasseur devait asseoir l'exploitation là où le taillis était le plus épais, sans dépeupler la forêt. Au Parc Sainte-Anne. ce furent les difficultés cle l'exploitation et de la vidange quii cngagèrent à renoncer à une exploitation régulière: la forêt fut néanmoins divisée en deux quartiers où les morts-bois seraient exploités alternativement au cours des 4 premières et 5 dernières années do

(46) Cf. Bibl. M. de Salins. M. n ${ }^{\circ}$ I28. Ordonnances des Souverains de Franche-Comté relatives aux Sauneries de Salins. Art. in de l'Ord. de Ph. I.OYTE.

(47) I2 engrognes valaient I gros, I2 gros I franc.

(48). De place en place.

(49) Aux quartiers de Germingney, Chissey, et Chateauroillaux situés au S. E. du massif, près du gué de la Roche. 
la rotation. Les dispositions des articles $3^{\text {I et }} 3^{2}$ de l'Ordonnance du 27 août I607 ne purent être exactement appliquées que dans les forêts des Grands bois de Vers et de la Faye de Montrond. Celle-ci, de grancle étendue, fut divisée en' trois quartiers pour que la fassure y fut perpétuelle. Aucun chêne ou chêneau, mort ou vif ne pouvait être compris dans ces exploitations.

Les dispositions qui prescrivaient qu'aucun chêne ne fut exploité sans avoir été préalablement marqué ne purent être d'ailleurs partout respectées. La Chènaie de la Fresse de Vers et de Montrivel n'était formée que de jeunes plançons et des cépées que les abus des usagers y avaient substituées. Le marqueur n'y pouvait désigner un à un tous les bois qu'il était utile de couper. Le règlement clisposa que le bois, divisé en trois quartiers, affectés chacun à une période triennale, serait parcouru à la rotation de 9 ans. Le fasseur y exploiterait les cépées, réserve faite du meilleur. rejet et les plançons là où ils étaient proches l'un rle l'autre de moins d'une toise sans cn pouvoir prendre plus d'un sur trois à chaque passage.

Le règlement du bois Mouchard, dont les dispositions généralcs furent rendues applicables à la plupart des chênaies et aux sapinières, par les règlements particuliers, prescrivait de ne marquer que les chênes secs ou escoulonnés sur le pied, ne portant plus paisson, sans attoucher aucunement aux chênes, pommiers et poiriers vifs. Les arbres rompus et déracinés devaient être également marqués. Au delà de ro par an, ils devaient être précomptés sur la possibilité. Le contrôle des exploitations serait tenu sur le registre du marqueur. Comme les chênes secs et escoulonnés étaient devenus rares au bois Mouchard, le nombre des arbres qu'on y pouvait marquer chaque année fut fixé à Ioo.

Le règlement des Grands bois de Vers où les chênes exploitables étaient aussi rares, et les conditions de végétation moins favorables, autorisa la marque de 45 chênes moyennement gros chaque année, et celui du Parc Sainte-Anne, situé sur le versant rocheux escarpé, de 8. Aucune exploitation régulière de chênes ne fut prévue à la Faye de Montrond qui en était mal peuplée, ni aux quartiers des bois de Chaux où les Sauneries n'étaient autorisées qu'à exploiter des mortsbois.

Dans les sapinières banales où les feuillus étaient rares, les règlements de l'Ordonnance du 8 juin I6r I ne réglèrent aucune exploitation de morts-bois. Celle des sapins ne devait porter que sur des bois secs et escoulonnés. Le règlement de la forêt de Combalerin ajoute cependant " qu'à leur défaut, pourront être marqués aucuns de moindre croissance et empêchant l'un l'autre, ce que toutefois on ne fera que par avis et participation du Juge et Officiers de notre Saunerie ". La possibilité des coupes annuelles était réglée à $60 \mathrm{sa-}$ pins pour Combalerin, 90 sapins médiocrement gros pour le Scay. $25^{\circ}$ sapins médiocrement gros pour le Jura, non compris $5^{\circ}$ sapins 
pour la fabrication des bosses (50) à prendre 30 au premier quartier de la forêt du Jura et 20 au premicr quartier de la forêt du Scay.

Comme les arbres secs et cscoulonnés étaient seuls tenus pour ('xploitables, les possibilités avaient été réglées en fonction du nonbre d'arbres surannés qu'on trouvait alors dans les chênaies et les sapinières dont le matériel avait été presqu'entièrement renouvelé au cours du siècle précédent, et qui, par conséquent, y étaient clevenus assez rares. Sans cioute, les clescriptions de Simon Musv attestent l'existence en 1602 dans plusieurs quartiers et singulièrement sur la moitié de la contenance du Jura, de sapinières assez riches, comprenant une proportion élevée d'arbres de la $\mathrm{I}^{\text {re }}$ espèce. Mais si les limites des trois dernières espèces de Simon Musy étaient assez étroites, celle de la $\mathrm{I}^{\text {re }}$ qui englobait tous les arbres susceptibles de donner des pièces d'une dimension supérieure à celle d'une panne étaient fort larges. La consistance même des peuplements oì les arbres de la $\mathrm{I}^{\mathrm{re}}$ espèce étaient nombreux montre assez qu'il. $\mathrm{y}$ en avait peu de vraiment vieux. Et si, en I6 I I, les rédacteurs de l'Ordonnance avaient trouvé le Jura très diminué, c'est parce que les très vieux bois y étaient devenus rares plutôt qu'en raison du délabrement des lisières du massif.

Pour maintenir la production de la sapinière de Combalerin. la plus proche de Salins, à un chiffre ralativement élevé. on avait dì $\mathrm{y}$ envisager l'exploitation de bois verts. Par contre, au Scay ct au Jura. bois secs et escoulonnés devaient suffire à l'application de la possibilité $(5 \mathrm{r})$. C'étaient soit des reliques des vieux peuplements lécrits en I.572 par Jean de Pomereulx (52) soit les débris de peuplements hien plus jeunes délabrés par les abus des riverains. En précisant que la possibilité des forêts de Scay et clu'Jura serait cl'ar. hres médiocrement $g r o s$. l'Orclonnance avait sans cloute entendlu mettre un terme à l'abus qui consistait à ne clélivrer au fermier que rle très gros bois. Lors de la visite de I602, Simon Musy s'était informé du rendement des sapins délivrés pour la fassure dans les forêts de Combalerin et du Scay de Chalamont. Dans la prenière, fort pauvre et où il n'y avait pas de chnix, il n'était que de I 500 fas-

(50) Tonneaux pour le transport des sels.

(5I) Voir supra la description du $3^{\mathrm{e}}$ Quartier de la forêt du Scay. On lit d'ailleurs dans le P. V. de visite de $\mathrm{I} 602$, à l'article Jura, une déclaration des commis du fermier portant « qu'ils n'entendaient point que l'on marque d'autres (sapins) que de secs pour ce qu'il y en avait si grand nombre dans ladite forêt qu'on ne les saurait épuiser de longtemps, avec ce qu'ils sont plus propres pour fassurer (réduire en fassins) et charroyer $\gg$.

(52) La survivance en I6I de reliques de vieux peuplements dont le délabrement était déjà commencé en $1_{542}$ ne doit pas surprendre. Dans la même forêt, les belles futaies régulières dont le développement suivit les abus de $l_{\text {a }}$ période révolutionnaire étaient toujours parsemées de vieux bois en I86r. (C.f. Arch. Conservation des E. et F. de Besançon. Aménagement de la forêt domaniale de Levier, I86r). 
sins (53) par arbre, dans la seconde de 2500 , soit environ io et I7 $\mathrm{m}^{3}$ par arbre, non compris les déchets de fabrication.

Les bois destinés à la confection des bosses avaient fait l'objet d'une possibilité spéciale à recruter tant au Scay qu'au Jura dans un quartier déterminé. Sans doute, y trouvait-on mieux qu'ailleurs les bois convenables, mais on doit noter également qu'au Jura ces bois dominaient une magnifique renaissance.

4. La sylviculture des Ordonnances. - Les Taillis. - La restauration des sapinières. - Les Futaies: la récolte des produits principaux, ses règles, lcur application aux chênaies et aux sapinières; la récolte des produits intermédiaires. - La genèse d'une sylviculture: de la culture du chêne à la culture du sapin.

L'Ordonnance du 27 août I607 avait consacré l'échec de la tentative poursuivie depuis plus d'un siècle par les Officiers de la Saunerie en vue d'introduire dans tous les taillis destinés à la cuite des muires le traitement en taillis régulier. Les dispositions qui, anciennement avaient limité la jouissance de l'usager dans les bois communs avaient par là-même défini son droit: il y pouvait couper à son gré les morts-bois, et y pratiquait en fait un furetage désordonné. Le champelage des morts-bois avait d'ailleurs été la condition du maintien et du développement des futaies de fruitiers et singulièrement des chênaies. Mais, lorsqu'aux environs de Salins, les bois taillis se multiplièrent par l'abandon de fait ou de droit des essences réservées. les usagers restèrent en possession du droit de les champeler ct ne s'en laissèrent pas clessaisir. Appliqué aux bois banaux. le traitemont en taillis régulier parait d'ailleurs n'avoir clomé au Xvi" siècle que des résultats assez décevants. Les besoins toujours pressants de la Saunerie ne permettaient pas d'attendre que le taillis ait atteint des dimensions suffisantes et l'exploitation tout à rez ne gas. pillait pas moins de bois que le champelage. Les fasseurs qui, au cours de la seconde moitié du $\mathrm{xvI}^{\boldsymbol{\theta}}$ siècle, reçurent des bois en nombre revinrent donc à la pratique du champelage. Mais n'ayant pas intérêt à discourir tout le bois comme les usagers. chacun coupant là où mieux lui convenait, ils tinrent néanmoins la laie en n'y exploitant que les bois de la climension exigée par la Saunenic. I'application de ce mode de traitement avait donné d'assez bons résultats pour que l'Ordonnance du 27 août I607 ait cru devoir l'ét:ndre à toutes les forêts banales.

Si les anciens usages. qui dans le principe avaient ignoré les bois taillis, avaient constitué un sérieux obstacle à toute tentative d'en uroaniser l'exploitation industrielle, on n'eut qu'à y puiser pour pour-

(53) En ISI I, le fassin a 6 pieds de long et la grosseur du bras. Son volume ne peut être estimé à moins de $0,0066 \mathrm{~m}^{3}$. 
voir au rétablissement des sapinières ruinées, sauf à prendre les mesures nécessaires pour que les moyens mis en nuvre soient effectiviment appliqués. Les dispositions des articles 45 à 52 de 1'Orclonnance du 22 septembre 1607 : mise en ban pour une durée de 20 ans de l'essence réservée, le sapin, dans les bois de la Joux, Maubelin, Loyaubault et Arc, interdiction d'en couper aucun à l'avenir sinon pour les besoins réels de l'usager et les emplois auxquels il était réservé, inspirées d'ailleurs des Articles de I 542 sont très exactement dans la tradition de la sylviculture médiévale.

Les articles de $\mathrm{I} 542$ n'avaient pas eu à traiter de la marque des futaies; c'était alors l'affaire des Officiers de la maison de Chalon. En 1607 , les rédacteurs des Ordonnances en formulèrent les règles. (Article I4 de l'Ordonnance du 27 août I607):

“ Ne pourrons marquer pour la fassure plus grand nombre de pieds qu'il n'est permis en chacun bois par le règlement sur çe fait ni autre que secs (et) escoulonnés, du moins aussi longtemps qu'il y aura tels, des lieux les plus peuplés, oires que plus éloignés, accessibles néanmoins pour la traite et charroi. Et à défaut desdits bois, ils ne pourront toucher aux vifs sinon par l'avis des Officiers de la Saunerie qui en ce cas pourvoiront que l'on ne marque sinon ceux de moins apparente croissance et empêchant celle des autres et se conformeront au surplus en tout et partout à ce qui est ordonré par icelui règlement $)$.

Les forêts où ces règles devaient être appliquées étaient, nonobstant quelques droits d'usage, et singulièrement l'usage aux mortsbois et au bois mort dans plusieurs chênaies, des forêts banales. Fn rroit, les arbres n'y étaient exploitables que parvenus au terme naturel de leur existence (54). Les arbres très dépérissants toutefois, les chênes qui ne pouvaient plus porter de glands par exemple, y purent être assez tôt assimilés aux arbres morts. Les requêtes présentées par les fasseurs du bois Mouchard et de Combalerin lors de la visite de I542, pour que de tels arbres fussent délivrés pour la fassure, semblent impliquer qu'ils étaient dès lors marqués par les officiers de Chalon, mais employés à d'autres fins. En I602, l'usage était de marquer les arbres escoulonnés comme les arbres morts pour la fassure. En principe, un arbre escoulonné était un arbre écimé ou $\mathrm{sec}$ en cime Le terme était cependant assez imprécis pour qu'on nit pu être tenté de l'appliquer à tout arbre suranné. En ı607, les rédacteurs des Ordonnances admirent en tous cas l'utilité d'élargir, lc cas échéant, le recrutement des possibilités, et autorisèrent qu'on y appliquât des arbres verts " de moins apparente croissance et em-

(54) Les bois morts étaient préférés aux bois vifs pour la fassure: « Combien que pour l'usage de la fassure il (le fasseur) aime beaucoup mieux ies secs que les verts, d'autant que les secs se fendent mieux et plus facilement et sont plus légers et propres à charroyer (P. V. de visite de I602. Art. Jura). Voir aussi note $5 \mathrm{r}$. 
pêchant celle des autres ") mais l'avis et la participation aux opérations des officiers de la Saunerie fut dans ce cas exigé.

De la domestication du porc au développement de l'industrie amorcé dès le Xıve siècle par la vulgarisation de la sidérurgie à deux temps (55), la production du gland avait été la fin incontestée de la culture du chêne et l'on conçoit que dans des peuplements voués à la production de fruits, les seuls arbres exploitables aient été ceux qui n'en pouvaient plus porter. La pratique d'une telle sylviculture entraînait nécessairement la constitution de peuplements irréguliers peu serrés, remarquables, sauf accidents ou abus, par la prédominance des vieux et très vieux bois (56). Bien que l'extraction d'un chêne ne portant plus paisson ait pu faciliter la croissance de quelques chêneaux dans la mesure où cette extraction anticipait la mort de l'arbre, la règle de porter la coupe aux lieux les plus épais n'était, appliquée à de telles formations, qu'une invitation à ne pas négliger les cantons éloignés, toujours moins fréquentés.

En I607, la mise en ban par les Princes de la Maison de Chalon d'une partie de leurs sapinières ne datait guère que de deux siècles. Sans doute, dès le haut Moyen-Age, la loi Gombette avait mis les essences résineuses au rang d'essences réservées (57). Mais il fallait que la Silva Jurensis fut colonisée et. en grande partie, défrichée pour qu'on songeât à en conserver les restes. A cet effet, on eut recours au procédé traditionnel de la mise en ban, et, de ce fait, les pratiques de la sylviculture qui assuraient depuis plusieurs millénaires le maintien des futaies de fruitiers et principalement des chênaies, se trouvèrent transportées dans les sapinières où appliquées à un tout autre objet, elles eurent de tout autres effets.

Alors que dans la chênaie une large trotée est nécessaire à l'installation d'un cône de régénération dont en fin de compte ne survivront que peu d'éléments qui s'incorporeront à la futaie, dans la sapinière, d'épaisses renaissances s'établissent sous les vieux peuplements existants et se développent au fur et à mesure de leur dislocation. C'est sur ces renaissances, dont elles assuraient le dégagement, que la coupe des arbres secs et escoulonnés des lieux les plus épais et, le cas échéant, de ceux de la moins apparente croissance et empêchant celle des autres devait être faite. Elle suivait la régénération, et loin d'entraver l'évolution naturelle de la sapinière vers des formes régulières, elle y aidait. Ainsi les peuplements mis en ban au Scay et au Jura qui dataient vraisemblablement du XIve siècle, après avoir vieilli en bloc, se disloquèrent au $\mathrm{xvI}^{\mathrm{e}}$ siècle. Cenx qui les remplacèrent alors disparurent au cours de la premic̀re moi-

(5.5) Où le fer est obtenu par l'intermédiaire de la fonte.

(56) De telles formations dont la ruine fut consommée en France par la mise en coupe réglée des futaics domaniales en août 1578 , puis par l'application de l'Ordonnance de 1669, ont survécu par places en Franche-Comté jusqu'au milieu du XVIII ${ }^{\mathrm{e}}$ siècle.

(57) Lois Gombette. Titre 38. 
tié du $\mathrm{XvII}^{\mathrm{e}}$ siěcle. Ni les abus de la période révolutionnaire, ni les exploitations de la première moitié du XIX siècle, ni les efforts des aménagistes depuis le second Empire n'ont encore rompu entièrement l'uniformité des peuplements de ces forêts.

Les coupes réglées par pieds d'arbres portaient exclusivement sur des bois parvenus à leur terme d'exploitabilité. Elles ne réalisaient donc que des produits principaux. Aujourd'hui, la récolte d'une forêt se fait autant de produits intermédiaires, d'arbres enlevés en vuc de favoriser la croissance des peuplements que d'arbres parvenus à le ur terme d'exploitabilité, bien qu'on ait renoncé à produire les très gros bois qui étaient alors recherchés. Les Ordonnances paraissent avoir négligé là une source importante de revenu.

A la clate de leur rédaction, la chênaie occupe toujours une place très importante dans l'écononie rurale comtoise (58). Le gland en demeure la production essentielle. Dans une chênaie à glands bien conservée, la récolte de produits intermédiaires n'était guère à considérer puisque son peuplement était formé d'arbres trapus à cimes bien développées où prédominaient les vieux et les très vieux bois. I.es chêneaux qui s'y installaient dans les trouées laissées par l'exploitation des arbres surannés étaient aussitôt dégagés pour être promptement amenés à fruit: " ains ne couperont jeunes chêneaux quand seront en apparence de croitre pour maisonner, mais seront tenus les dits fasseurs de les garder ct décombrer au pied pour les faire (roitre " (59). La conduite de l'opération est ici laisséc au fasseur qui lexécutait tout en exploitant les morts-bois. Mais dans les chênaies clévastées, cette opération avait pris une telle importance que le: rédacteurs des Articles de I5 22, au bois Mouchard, et de 1'Ordonnance de r6rr à la Fresse jugèrent nécessaire de la régler. Tes articles de 5.5 .2 autorisèrent la coupe des chêncaux " moyennant qu'il en demeure toujours des plus beaux à unc toise près l'un de l'autre, ainsi que le bois le pourra porter ». Fin fait, cette dispocition. permettait au fasseur de couper tous les chênes situés à moins d'une toise du chêne voisin. De même, l'Ordonnanc de r6 i I autorisa la coupe des chênes qui seraient plus près l'un de l'autre (que la longueur d'une toise, interdisant toutefois qu'on en coupât plus d'un de trois à chaque passage. Le nombre des chêneaux ainsi conservés était fort éloigné de celui qu'on aurait obtenu par une plantation régulière à I toise d'intervalle, vraisemblablement très inférieur à t 000 à l'hectare aux lieux les plus épais. Compte tenu d@s accidente à prévoir et de l'activité des délinquants, c'était le moins qu'on en pût laisser pour reconstituer la chênaie.

Plus encore que les chênaies, les sapinières avaient à souffrir du

(58) Cf. Les sources de la Sylviculture de Maclot. Introduction. Rev. for. franc., avril I95I, p. 295.

(59) Cf. Arch. Doubs. Chambre des Comptes B. 240. Mandement du II janvier $1535 / 6$ dressé par les officiers de Chalon. Art. 20. 
pillage des riverains (60). Le problème de l'éclaircie de peuplements léjà trop clairs ne s'y posait pas, mais bien celui de leur protection. L'article I4 de l'Ordonnance du 27 août r6o7, en autorisant la coupo des arbres de moins apparente croissance et empêchant celle des autres, avait néanmoins formulé un principe susceptible d'être appliqué à la récolte de produits intermédiaires dans les peuplements en voie de croissance, et, de fait, il suffira que le peuplement d'un quartier du Jura devienne trop épais pour qu'on décide de l'éclaircir (6o bis).

Bref, si la pratique régulière de l'éclaircie dans les chênaies et sapinières rendues déjà trop claires par l'activité des délinquants était impensable en 1607 , de telles opérations pouvaient être et étaient effectivement prescrites par des règlements spéciaux lorsqu'elles étaient reconnucs nécessaires à la formation ou au développement des peuplements.

Bien que rédigées pour être appliquées anx chênaies comme aux sapinières, les règles de culture formulées par l'article I4 de l'Ordonnance du 27 aồt 1607 constituaient en fait une adaptation à la culture des sapinières des règles de culture traditionnelles de la chênaie. Jusqu'à la mise en ban par les Seigneurs de Chalon- Irlay d'ume partie de leurs sapinières, il n'existait pas de sylviculture du sapin. La forêt, tenue pour une source inépuisable de produits ligneux indispensables, mais sans autre prix que l'air ou l'eau, n'était cultivée que pour ses fruits. Mais dans la région de Salins, le développement de l'agriculture et de l'industrie et l'épuisement parallèle des. ressourccs forestières ont, dès le XIV siècle, fait du bois un matériau apprécié. à la production duquel il est devenu intéressant de veiller. Tes tabous qui depuis plusieurs millénaires avaient permis l'établissement et la conservation des chênaies, pouvaient. appliqués aux es-ences résineuses, reconstituer et enrichir les sapinières, mais, $a$ but atteint, ils ne permettaient qu'une utilisation très médiocre (ce peuplements oi il n'était nas question de produire les fruits. mais du hois. Aussi. lorsque les vieux peuplements des sapinières miscs en ban se disloquèrent au $\mathrm{xvI}^{\mathrm{e}}$ siècle, l'élargissement de la récolte des bois morts aux bois escoulonnés et aux bois de moins apparente croissance et empêchant celle des autres dans la mesture nécessaire à la satisfaction des besoins, et sa concentration aux lieux les plus épais, c'est-à-dire sur les renaissances en pleine croissance (lu'il convenait re clégager, s’imposa tant pour la conservation des massifs que pour l'augmentation de leur rendenient clans toute la mesura compatible avec la production des très gros bois, à traiter par la fente. alors recherchés pour la Saunerie.

Cette sylviculture du sapin où l'action de l'homme toujours com-

(6o) Cf. supra les descriptions de forêts et les statistiçues nécessairament très incomplètes des bois coupés en délit.

(6o bis) Cf. ci-après: L'Application des Ordonnances. 
mandée par l'évolution naturelle des peuplements se borne à y aider en en anticipant les effets, se situe aux antipodes de son homonyme contemporain, le jardinage cultural. Celui-ci, cn effet, est à la pointe d'un art forestier idéaliste, qui, loin de se soumettre à la nature, entend la dominer et conduire les peuplements au pas de l'oie vers des normes abstraites (6I). L'art réaliste des Officiers de la Sa11nerie n'est qu'un art primitif, sans habiletés et sans perspective. fonlé sur l'exacte observation des faits et le bon sens.

\section{Application des Ordonnances.}

L'application des Ordonnances fut sérieusement entravée par les guerres qui, de 1636 à I646, ruinèrent et clépeuplèrent la FrancheComté. Lorsqu'en I653 Daniel Privé et Etienne Pierre, conseiller et auditeur à la Cour des Comptes de Dole visitèrent avec Jacques Cugnot, juge à la Saunerie, les bois affectés à la cuite des muires pour y restaurer l'ordre que les Ordonnances y avaient établi, ils les trouvèrent sans forestiers, faute d'en pouvoir recruter, quelquesuns sans fasseurs, mais peu endommagés par les riverains devenus assez rares

Le procès-verbal de leurs travaux en date des 28 mai - 28 juin I653 (62) fait ressortir des retards souvent importants dans l'exploitation des taillis. Du côté des chênaies, le bois Mouchard est bien peuplé et en bon état. A la Fresse où la fassure est interrompue depuis I7 ans, les chênes, par places fort éloignés les uns des autres, sont noueux et tordus, mais le taillis abonde bien qu'il y en ait quantité d'exploité pour le chauffage des usagers et la fabrication de cercles pour les bosses. Cependant le sapin a pénétré clans la forêt où il s'avance du sud au nord. On le trouve en quantité notable dans la Fresse de Montrivel et on en signale quelques pieds dans la Fresse de Vers.

L'enrésinement des bois, où le rétablissement des sapinières avait été décidé en I 542, s'est rapidement poursuivi. Il est acquis à Loyaubault. A Maubelin, la sapinière couvre les confins de Scay et le quartier revendiqué par les habitants de Levier; ailleurs, l'enrésinement s'est trouvé entravé par l'abandon des exploitations usagères qui dégageaient systématiquement les sapineaux et le sapin resto disséminé dans les taillis devenus impénétrables. La majeure partie de la Joux est toujours peuplée de taillis, mais le sapin y a fait des progrès considérables au S.-E. du massif dans le triangle formé par les sapinières toujours conservées de Combalerin, dı Sauget et

(6I) Que les normes soient établies en fonction de lois statistiques ne change rien à l'affaire. Ces lois ne sont vérifiées qu'à l'échelle d'observation d'un très grand nombre de forêts dont les normes traduisent l'histoire moyenne. Chaque forêt a son histoire propre, ses peuplements ne peuvent être assujettis à de telles normes sans que leur développement naturel soit altéré.

(62) Cf. Arch. Doubs. Chambre des Comptes B. 2 II3 
des Lezinnes. Les taillis dont Arc est toujours peuplé sont analogues à ceux de Maubelin; les usagers commencent à y faire quelques abattues et à les champeler du côté de Villeneuve; les sapins qu'on y trouve sont fort éloignés les uns des autres et tout au plus propres à faire chevrons; les plus gros en ont été tirés " après les malheurs des guerres pour la nécessité des usagers ». Lors de la visite de $1658 / 59$ (63), on reconnaitra l'utilité d'y interdire à nouveau la coupe des sapins pour la durée de 20 ans " afin qu'ils puissent prendre une croissance convenable et telle qu'on puisse s'en servir utilement ». Le procès-verbal de visite de I653 signale également d'importants progrès du sapin dans le bois de Vignory. Mais comme il n'y était pas protégé par les Ordonnances, l'amodiataire des Sauneries le faisait réduire en quartelage.

Enfin, les sapinières banales furent trouvées bien peuplées et en bon état. Leur exploitation était d'ailleurs très ralentie depuis I7 ans. Elle était même suspendue à Combalerin. Au Scay, on fabrique cncore quelques fassins et des bosses. Au Jura. on ne fabrique plus que des bosses. Les bois qui y sont employés doivent être d'ailleurs exploités en dehors du quartier désigné à cet effet en 16r I où les bois convenables font défaut depuis 20 ans. L'extraction de ces très gros arbres y avait été pratiquée sans endommager la belle renaissance décrite en 1602. "Le bois est tellement peuplé en cet en(lroit », lit-on dans le procès-verbal de visite de 1653, "qu'il a été jugé utile de l'éclaircir et abattre une partie des moindres sapins commè cle cinq l'un afin que les autres prennent plus facilement leur croissance ). A défaut de s'être haussé jusqu'à une connaissance thénrique de l'éclaircie, les Officiers de la Saunerie avaient du moins une connaissance empirique des peuplements qui leur permettait die les éclaircir quand et comme il le fallait.

Quelques années plus tard, les deux conquêtes françaises et l'annexion de la Franche-Comté ouvraient un nouveau chapitre de l'histoire des bois affectés à la cuite des muires des Salines de Salins.

L. Turc.

(64) Cf. Arch. Doubs. Chambre des Comptes B. 2ri4. 